\title{
Developments in Primary Aldosteronism Subtyping Using Steroid Profiling
}

\author{
Authors \\ Taweesak Wannachalee', 2(D, Adina F. Turcu ${ }^{1}$ (D) \\ Affiliations \\ 1 Division of Metabolism, Endocrinology, and Diabetes, \\ University of Michigan, Ann Arbor, MI, USA \\ 2 Division of Endocrinology and Metabolism, Siriraj \\ Hospital, Mahidol University, Bangkok, Thailand
}

\author{
Key words \\ primary aldosteronism, adrenal vein sampling, steroids, \\ adrenal, aldosterone \\ received $\quad 28.12 .2019$ \\ accepted $\quad 09.03 .2020$ \\ Bibliography \\ DOI https://doi.org/10.1055/a-1141-3526 \\ Published online: 24.4.2020 \\ Horm Metab Res 2020; 52: 373-378 \\ (c) Georg Thieme Verlag KG Stuttgart · New York \\ ISSN 0018-5043 \\ Correspondence \\ Adina F. Turcu MD, MS \\ Division of Metabolism, Endocrinology, and Diabetes \\ University of Michigan \\ 1150 W Medical Center Drive, MSRB II, 5570B \\ 48109 Ann Arbor MI \\ USA \\ Tel.: +1 734647 8906, Fax: + 17342324839 \\ aturcu@umich.edu
}

\begin{abstract}
Adrenal venous sampling is the standard of care for identifying patients with unilateral primary aldosteronism, which is often caused by an aldosterone producing adenoma and can be cured with surgery. The numerous limitations of adrenal venous sampling, including its high cost, scarce availability, technical challenges, and lack of standardized protocols, have driven efforts to develop alternative, non-invasive tools for the diagnosis of aldosterone producing adenomas. Seminal discoveries regarding the pathogenesis of aldosterone producing adenomas made over the past decade have leveraged hypotheses-driven research of steroid phenotypes characteristic of various aldosterone producing adenomas. In parallel, the expanding availability of mass spectrometry has enabled the simultaneous quantitation of many steroids in single assays from small volume biosamples. Steroid profiling has contributed to our evolving understanding about the pathophysiology of primary aldosteronism and its subtypes. Herein, we review the current state of knowledge regarding the application of multi-steroid panels in assisting with primary aldosteronism subtyping.
\end{abstract}

\section{Introduction}

Primary aldosteronism (PA) is the most common form of secondary hypertension, affecting 5-10\% of all hypertensive patients [13 ] and up to $20 \%$ of those with resistant hypertension [4, 5]. PA is associated with higher prevalence of metabolic syndrome, and disproportionately higher cardiovascular and renal morbidity and mortality than essential hypertension [6-10]. Early diagnosis of PA and implementation of targeted therapy are, thus, essential for reducing its associated complications [6, 11, 12]. Conventionally, PA has been classified into two major subtypes: bilateral hyperaldosteronism (BHA), and unilateral PA. Unilateral PA accounts for $30-50 \%$ of PA cases, and it is commonly caused by an aldosterone-producing adenoma (APA) [3]. APAs are ideally treated with laparoscopic adrenalectomy, which often cures PA and alleviates the cardiovascular morbidity in these patients $[6,7,10]$. Converse- ly, patients with $\mathrm{BHA}$ require life-long medical therapy that typically includes a mineralocorticoid receptor antagonist (MRA) [13].

Unlike other disorders of adrenal hormonal excess, the source(s) of aldosterone excess in PA cannot be reliably identified by conventional imaging studies. Two major factors contribute to the poor performance of adrenal imaging in PA subtyping: 1) areas producing aldosterone excessively can be smaller than the threshold for detection by cross-sectional imaging, or they might not distort the adrenal cortex anatomy at all [14] and 2) the prevalence of nonfunctional adrenal cortical nodules increases with age, as does that of PA $[15,16]$. Indeed, CT findings can lead to erroneous subtyping in up to $50 \%$ of PA cases [17-20]. Consequently, adrenal vein sampling (AVS) is endorsed by expert guidelines as the only reliable tool for PA subtyping [3, 21, 22]. AVS, however, is a costly, invasive, and operator-dependent procedure, with scarce availability. Further- 
more, many aspects regarding AVS protocols and data interpretation remain controversial and differ among institutions [23, 24].

Recent progress in the understanding of PA pathogenesis has driven efforts to develop non-invasive tools for PA subtyping. One such approach is identifying steroid biomarkers pathognomonic of APAs. The transition from immunoassays to mass spectrometry has facilitated the simultaneous measurement of multi-steroid panels in small volume biospecimen samples. Steroid fingerprints specific to patients with APAs measured in peripheral blood could eliminate the need for AVS in all other PA patients, who are best served by medical therapy. Herein, we review the recent advances in the application of steroid mass spectrometry for PA subtyping.

\section{Steroid Profiles Characteristic of APAs with Different Underlying Aldosterone-Driver Somatic Mutations}

With the implementation of next-generation sequencing (NGS), a series of APA tissue studies conducted over this past decade have identified several somatic mutations in genes with impact on aldosterone synthesis: KCNJ5, which encodes the Kir3.4 (GIRK4) potassium channel; ATP1A1, which encodes the $\mathrm{Na}^{+} / \mathrm{K}^{+}$ATPase $\alpha-1$ subunit; ATP2B3, encoding a Ca ${ }^{2+}$ ATPase 3; and CACNA1D, which encodes a voltage-dependent L-type calcium channel subunit 1D [25-28]. Such mutations account for at least $90 \%$ of sporadic APAs $[29,30]$, and they all facilitate inappropriate intracellular calcium entrance, which subsequently boosts aldosterone production.

Detailed histopathological studies of APAs have revealed morphologic and enzymatic heterogeneity across genotypes (๖ Fig. 1). APAs harboring KCNJ5 mutations are typically composed of large cells, with clear, lipid-rich cytoplasm, resembling zona fasciculata (ZF) cells [31-33]. Conversely, more variability has been reported in the histological features of APAs with other underlying somatic mutations [31, 34, 35]. Overall, APAs harboring ATPase or CACNA1D mutations display a higher proportion of compact eosinophilic, or lipid-poor, cells, similar to zona glomerulosa (ZG) cells (> Fig. 1) [34]. Other features that distinguish APAs with KCNJ5 mutations include their larger size and higher expression of CYP11B1 and CYP17A1 $[34,36,37]$. The co-localization within the same tumor of aldosterone synthase (CYP11B2), which is normally restricted to the ZG, and $17 \alpha$-hydroxylase/17,20-lyase (CYP17A1), which is expressed in the ZF and zona reticularis, allows these KCNJ5-mutatated APAs to produce "hybrid steroids", such as 18-hydroxycortisol (18OHF) and 18-oxocortisol (18oxoF, > Fig. 2) [38, 39].

Evidence to support the previously suspected capability of $K C$ NJ5-mutated APAs to over-produce hybrid steroid has only recently emerged. In a study of 79 patients with APAs of various genotypes (27 KCNJ5, 9 ATPase, 7 CACNA1D, and 36 "wild-type"), Williams et al. found that patients with APAs harboring KCNJ5 mutations had abundant concentrations of $180 x o \mathrm{~F}$ in peripheral venous plasma: 21 -fold higher compared to the wild-type group $(p<0.05)$ and 16 -fold higher compared to all other groups combined ( $p<0.0001)$. Patients with APAs harboring ATPase mutations displayed the highest peripheral concentrations of aldosterone, cortisol, 11-deoxycorticosterone (DOC) and corticosterone.

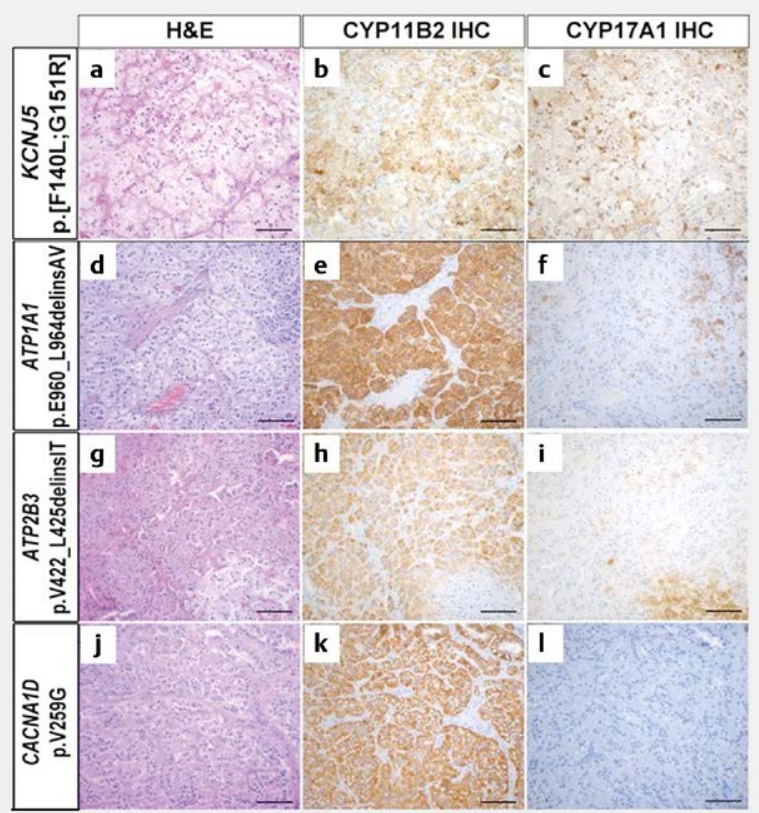

- Fig. 1 The histopathologic characteristics in APAs with different underlying aldosterone-driver somatic mutations. Figure reproduced from reference [30]. H \& E: Hematoxylin and eosin; IHC: Immunohistochemistry [rerif].

Conversely, patients with CACNA1D-mutated APAs had lower concentrations of aldosterone, corticosterone, and DOC relative to all other groups combined. A panel of 7 steroids measured in peripheral plasma correctly classified $92 \%$ of APAs based on their underlying mutations [40]. A limitation of this initial study was that the areas selected for DNA sequencing were not identified with CYP11B2 immunostaining, and consequently, $45.6 \%$ of the APAs included were presumed to be "wild type." Subsequent studies that implemented CYP11B2 immunohistochemistry to guide the selection of tissue for NGS have shown that somatic mutations can be found in up to $90 \%$ of APAs $[29,30,41]$. The incomplete stratification of APAs by mutation status has likely diluted the power of genotype-specific steroid signatures associated with APAs.

Further refining the steroid fingerprints of APAs according to their underlying pathology could be made possible by larger studies that include sufficient numbers of patients with APAs that harbor rare mutations. For instance, recent evidence suggests that ATP2B3 mutations lead to distinct histopathological features [34] and PA phenotypes from those with ATP1A1 mutations. In a study that included 61 patients with known APA mutation status (24 KCNJ5, 22 CACNA1D, 10 ATP1A1, 4 ATP2B3, and 1 (TNNB1), we found that peripheral venous aldosterone concentrations were higher in patients with ATP2B3 and KCNJ5 mutations than in those with ATP1A1 or CACNA1D mutations [42]. In-depth understanding of the molecular mechanisms and histopathology of APAs will further leverage the development of non-invasive diagnostic tools. 


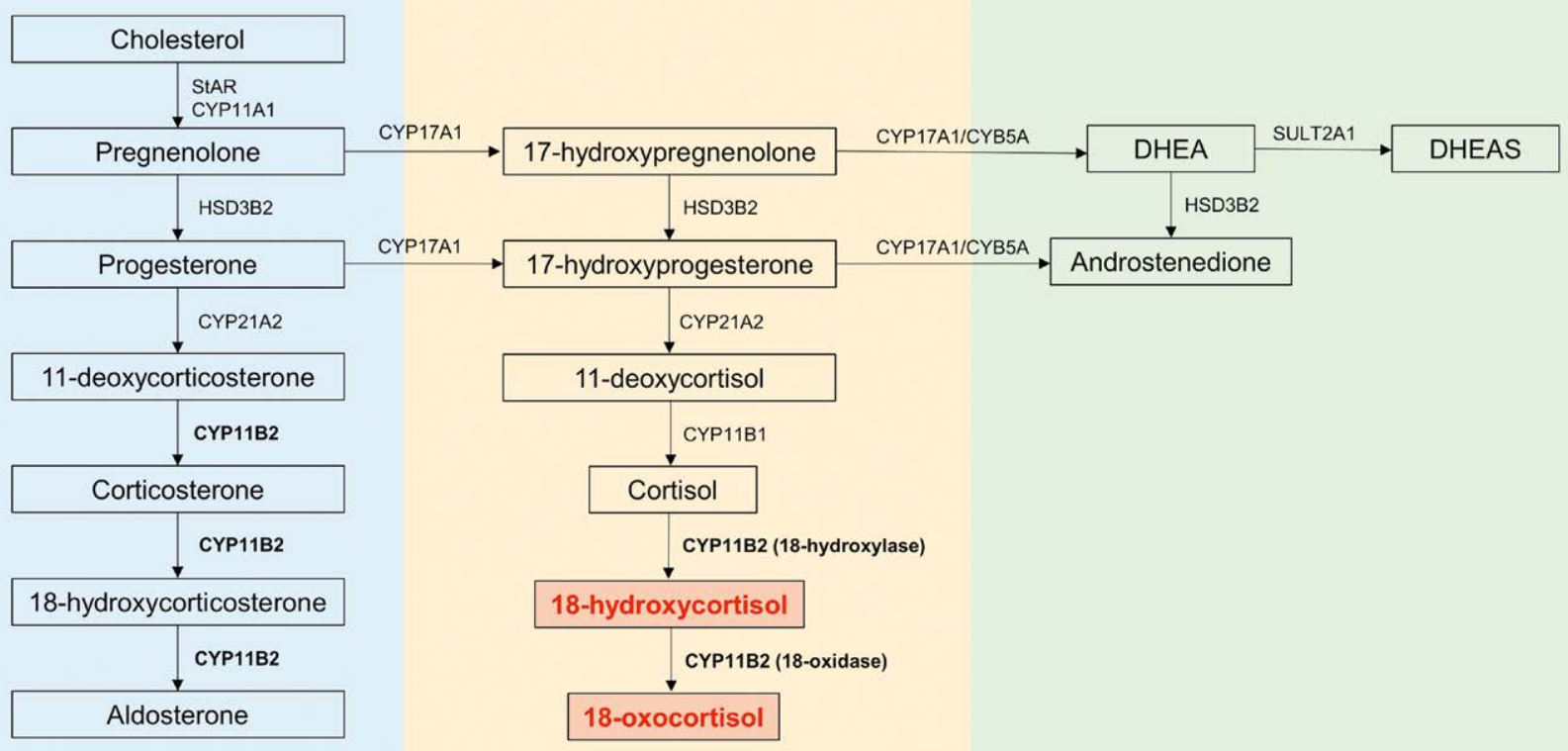

Zona glomerulosa

Zona fasciculata

Zona reticularis

- Fig. 2 Adrenal steroidogenesis, illustrating the synthesis of "hybrid steroids", which require both 17 $\alpha$-hydroxylase/17,20-lyase (CYP17A1), which is expressed in the zona fasciculata (ZF) and reticularis (ZR), and aldosterone synthase (CYP11B2), which is expressed in the zona glomerulosa (ZG). DHEA: Dehydroepiandrosterone; DHEAS: Dehydroepiandrosterone sulfate; HSD3B2: 33-Hydroxysteroid dehydrogenase type 2; SULT2A1: Sulfotransferase type 1A; CYB5A: Cofactor cytochrome $b_{5}$; StAR: Steroidogenic acute regulatory protein; CYP11A1: Cytochrome P450 cholesterol side-chain cleavage; CYP11B1: 11ß-Hydroxylase; CYP21A2: 21 $\alpha$-Hydroxylase.

\section{Steroid Profiling as a Tool for PA Subtyping}

Efforts to identify biomarkers specific to APAs preceded the discovery of aldosterone-driver mutations and characteristic histological features associated with these tumors. Early studies noted elevations of $18 \mathrm{OHF}$ and $180 \mathrm{oxoF}$ in patients with PA as compared to those with essential hypertension $[43,44]$, and particularly so in APA versus BHA [45-47]. The production of $180 x \mathrm{oF}$ and $18 \mathrm{OHF}$ was recognized to require shared enzymatic features of the $\mathrm{ZG}$ and $\mathrm{ZF}$ ( Fig. 2) [48] as was observed in glucocorticoid remediable aldosteronism [49]. As shown more recently, APAs harboring KCN/5 mutation, which display such histological features, are globally the most common type of APAs.

In a Japanese study of 234 PA patients (113 with APA) LC-MS/ MS was used to compare the peripheral concentrations of hybrid steroids and aldosterone between PA subtypes [50]. Both $180 x \mathrm{oF}$ and $18 \mathrm{OHF}$, as well as aldosterone were significantly higher in patients with APA than in those with BHA. Receiver operating characteristic (ROC) curve analyses showed that an $180 x 0 F$ peripheral venous concentration of $4.7 \mathrm{ng} / \mathrm{dl}$ had a sensitivity of 0.83 and specificity of 0.99 to discriminate APA from BHA. Aldosterone and $18 \mathrm{OHF}$ were also highly accurate for distinguishing between the two groups [area under the curve (AUC) of 0.917 and 0.85 , respectively] [50].

In the subsequent year, Eisenhofer et al. published their findings from 216 European patients with PA (126 APA and $90 \mathrm{BHA}$ ) in whom 15 steroids were measured by LC-MS/MS [51]. Although peripheral venous $180 x 0 F$ was on average 8.5 -fold higher in patients with APA than those in BHA, significant overlap between the two groups limited the discriminatory power of $180 x 0 F$ (AUC, 0.659). A panel incorporating all 15 steroids measured, however, achieved an AUC of 0.889 for distinguishing APA from BHA [51].

Racial differences in the prevalence of aldosterone-driver somatic mutation and genotype-specific steroid profiles in patients with APAs contribute to the variability in performance of steroid biomarkers for PA subtyping. As we have learned over the recent years, KCNJ5 mutations are found in up to $80 \%$ of East Asians populations [52,53], but in only roughly $40 \%$ of Europeans, Australians, and white Americans, who display a wider spectrum of APA genotypes [30,54-58]. Notably, although somatic CACNA1D mutations are overall the most frequent in African American patients with APAs (43\%), KCNJ5 mutations have the highest prevalence in women, regardless of race ( $55 \%$ of African American and $70 \%$ of Caucasian women) $[29,30]$. Considering that KCNJ5 mutated-APAs have the highest enzymatic capability to produce $180 x \mathrm{or}$ and $18 \mathrm{OHF}[38,39]$, these hybrid steroids are expected to be highly accurate in identifying patients with APAs among Asians and women with PA.

A major challenge in establishing reliable biomarkers that can distinguish APA from BHA is the lack of a precise gold standard for PA subtyping. Unlike the explosion of knowledge derived from patients with APAs over the past decade, the pathogenesis of BHA is less well understood. As patients with BHA rarely undergo surgery, 
the availability of tissue is scarce. A recent study of BHA adrenal tissue revealed that most such patients harbor micro-APAs and that $58 \%$ of these micro-APAs have CACNA1D mutations [59].

In vivo, PA subtyping has been dependent on AVS. Considering that AVS protocols around the world are not uniform, it is conceivable that PA subtype classification is center-dependent, particularly for intermediate cases. Potential sources of variability in PA subtyping based on AVS include use of cosyntropin, differences in AVS data interpretation, and concomitant glucocorticoid excess [60]. Data from referral centers that perform AVS both prior to and after stimulation with cosyntropin have shown that subtyping can be discordant between the two protocols in approximately a quarter of PA patients [42, 61]. Notably, in a study of 222 PA patients who underwent AVS at the University of Michigan and had successful catheterization both pre- and post-cosyntropin stimulation [42], the proportion of patients with discordant AVS subtyping based on pre- and post-cosyntropin AVS results was higher in African American than in Caucasian patients ( 45 vs. $17 \%, p=0.003$ ). African American patients with APAs commonly harbor CACNA1D mutations [29]. We found that, overall, patients with CACNA1D-mutated APAs had the least predictable impact of cosyntropin on the lateralization index [42], which could explain, at least in part, the higher proportion of discordant AVS results in this population. Stratified PA subtyping combining AVS results from both pre- and post-cosyntropin stimulation, revealed that cases consistently classified as unilateral had the highest adrenal vein and peripheral aldosterone concentrations, and the most profound contralateral suppression [42]. As the impact of cosyntropin on autonomous vs. normal aldosterone-producing cells remains elusive, cases with inconsistent lateralization might represent either small APAs or asymmetrical BHA. Other surrogates for aldosterone dominance derived from AVS data, such as the lateralization index and contralateral suppression index, have been relatively inconsistent as predictors of postoperative outcomes.[62-64] The lack of standardized criteria for adrenalectomy and only recent introduction of uniform postoperative follow up approaches [13] have further hampered our understanding of the spectrum of PA subtypes.

In a recent study, we used LC-MS/MS to compare the steroid profiles between stratified PA subtypes [65]. Out of 103 patients with valid AVS data both prior to and following cosyntropin administration, 20 patients met criteria for unilateral PA only at baseline and other 14 patients showed lateralization only after cosyntropin stimulation. Both baseline and cosyntropin-stimulated aldosterone and $180 x$ o concentrations were highest in the peripheral serum of patients consistently classified as unilateral. Discriminant analysis based on all 17 steroids measured in peripheral serum demonstrated robust separation of patients with consistent subtyping regardless of cosyntropin use, with intermediate zones represented by cases with discrepant lateralization results ( $\mathbf{F i g} \mathbf{3}$ ). Indeed, even with multi-steroid panels measured in peripheral blood samples, the distinction between cases classified as unilateral or bilateral based on baseline AVS data alone was poor (AUC of $0.586)$. In contrast, the same 17 -steroid panel measured in peripheral blood performed better in distinguishing patients with consistent unilateral or bilateral PA subtyping results based on pre- and

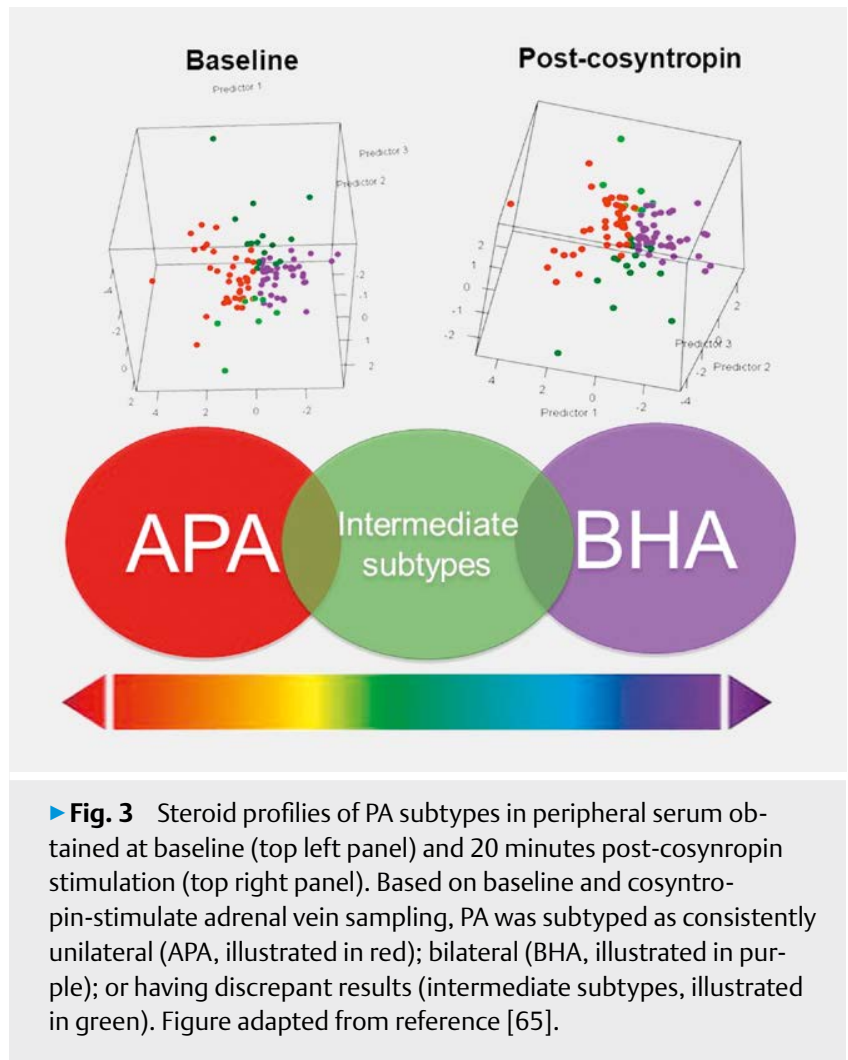

post-cosyntropin stimulation AVS data (AUC of 0.768 with steroids measured at baseline, and 0.907 with steroids measured after cosyntropin-stimulation) [65]. Our results emphasize that PA subtypes are difficult to dichotomize ( $\vee$ Fig. 3 ), and that AVS offers only a snapshot of the relative contribution of the two adrenal glands to aldosterone production. Long-term follow up of ambiguous PA cases is likely to inform about their evolution towards unilateral dominance of aldosterone excess or BHA.

While the development of steroid profiling for assisting with PA subtyping is still in its infancy, several aspects will mandate careful consideration as this tool evolves. Initial stages include establishing reliable reference ranges for steroids where such data is lacking, including for hybrid steroids, harmonization of standards, and quality assurance strategies. Incorporation of multi-steroid assays also adds complexity in results interpretation, possibly affecting the practical utility of multi-steroid panels. Although machine learning technology is frequently applied in research, designing simple interpretation algorithms will be of utmost importance for the busy clinician. Such algorithms might incorporate patients' demographics, other laboratory parameters (such as serum potassium), and/or imaging studies. Considering the numerous individual aspects to clinical care, it is likely that clinical decision making will use available tools complementarily. Finally, well-designed prospective studies, with randomization of treatment, and standardized long-term follow up, will be critical for establishing the utility of steroid profiling in PA subtyping and, hopefully, even in predicting postoperative outcomes. 


\section{Conclusions}

The development of non-invasive and widely available tools for PA subtyping is essential for leveraging personalized care and for maximizing the number of cured PA patients. Such innovations have been bolstered by major discoveries relevant to the pathogenesis and histopathological features of APAs emerged during the past decade. Steroids characteristic of APAs with KCNJ5 mutations will be particularly relevant in Asians of both sexes and in Caucasian women with sporadic APA. Refining the steroid fingerprints of APAs with other underlying mutations and elucidating the pathogenesis of BHA will eventually increase the accuracy of peripheral blood tests for PA subtyping and will thus circumvent the need for AVS in a large number of PA patients.

\section{Funding}

AFT was supported by grants1K08DK109116 from the NIDDK and 2019087 from the Doris Duke Charitable Foundation.

\section{Conflict of Interest}

The authors declare that they have no conflict of interest.

\section{References}

[1] Hannemann A, Wallaschofski $\mathrm{H}$. Prevalence of primary aldosteronism in patient's cohorts and in population-based studies - a review of the current literature. Horm Metab Res 2012; 44: 157-162

[2] Monticone S, Burrello J, Tizzani D et al. Prevalence and clinical manifestations of primary aldosteronism encountered in primary care practice. J Am Coll Cardiol 2017; 69: 1811-1820

[3] Funder JW, Carey RM, Mantero F et al. The management of primary aldosteronism: Case detection, diagnosis, and treatment: An Endocrine Society Clinical Practice Guideline. J Clin Endocrinol Metab 2016; 101: 1889-1916

[4] Calhoun DA, Nishizaka MK, Zaman MA et al. Hyperaldosteronism among black and white subjects with resistant hypertension. Hypertension 2002; 40: 892-896

[5] Douma S, Petidis K, Doumas M et al. Prevalence of primary hyperaldosteronism in resistant hypertension: A retrospective observational study. Lancet 2008; 371: 1921-1926

[6] Hundemer GL, Curhan GC, Yozamp N et al. Cardiometabolic outcomes and mortality in medically treated primary aldosteronism: A retrospective cohort study. Lancet Diabetes Endocrinol 2018; 6: 51-59

[7] Hundemer GL, Curhan GC, Yozamp N et al. Incidence of atrial fibrillation and mineralocorticoid receptor activity in patients with medically and surgically treated primary aldosteronism. JAMA Cardiol 2018; 3: 768-774

[8] Mulatero P, Monticone S, Bertello C et al. Long-term cardio- and cerebrovascular events in patients with primary aldosteronism. J Clin Endocrinol Metab 2013; 98: 4826-4833

[9] Fallo F, Veglio F, Bertello C et al. Prevalence and characteristics of the metabolic syndrome in primary aldosteronism. J Clin Endocrinol Metab 2006; 91: 454-459

[10] Hundemer GL, Curhan GC, Yozamp N et al. Renal outcomes in medically and surgically treated primary aldosteronism. Hypertension 2018; 72: 658-666
[11] Rossi GP, Cesari M, Cuspidi C et al. Long-term control of arterial hypertension and regression of left ventricular hypertrophy with treatment of primary aldosteronism. Hypertension 2013; 62: 62-69

[12] Iwakura Y, Morimoto R, Kudo M et al. Predictors of decreasing glomerular filtration rate and prevalence of chronic kidney disease after treatment of primary aldosteronism: Renal outcome of 213 cases. J Clin Endocrinol Metab 2014; 99: 1593-1598

[13] Williams TA, Lenders JWM, Mulatero P et al. Primary aldosteronism surgery outcome i. Outcomes after adrenalectomy for unilateral primary aldosteronism: An international consensus on outcome measures and analysis of remission rates in an international cohort. Lancet Diabetes Endocrinol 2017; 5: 689-699

[14] Nanba AT, Nanba K, Byrd JB et al. Discordance between imaging and immunohistochemistry in unilateral primary aldosteronism. Clin Endocrinol 2017; 87: 665-672

[15] Fassnacht M, Arlt W, Bancos I et al. Management of adrenal incidentalomas: European Society of Endocrinology Clinical Practice Guideline in collaboration with the European Network for the Study of Adrenal Tumors. Eur J Endocrinol 2016; 175: G1-G34

[16] Mantero F, Terzolo M, Arnaldi G et al. A survey on adrenal incidentaloma in Italy. Study Group on Adrenal Tumors of the Italian Society of Endocrinology. J Clin Endocrinol Metab 2000; 85: 637-644

[17] Williams TA, Burrello J, Sechi LA et al. Computed tomography and adrenal venous sampling in the diagnosis of unilateral primary aldosteronism. Hypertension 2018; 72: 641-649

[18] Lim V, Guo Q, Grant CS et al. Accuracy of adrenal imaging and adrenal venous sampling in predicting surgical cure of primary aldosteronism. J Clin Endocrinol Metab 2014; 99: 2712-2719

[19] Young WF, Stanson AW, Thompson GB et al. Role for adrenal venous sampling in primary aldosteronism. Surgery 2004; 136: 1227-1235

[20] Kempers M], Lenders JW, van Outheusden L et al. Systematic review: Diagnostic procedures to differentiate unilateral from bilateral adrenal abnormality in primary aldosteronism. Ann Intern Med 2009; 151: 329-337

[21] Young WF Jr. Diagnosis and treatment of primary aldosteronism: Practical clinical perspectives. J Intern Med 2019; 285: 126-148

[22] Rossi GP, Auchus R], Brown M et al. An expert consensus statement on use of adrenal vein sampling for the subtyping of primary aldosteronism. Hypertension 2014; 63: 151-160

[23] Monticone S, Viola A, Rossato D et al. Adrenal vein sampling in primary aldosteronism: Towards a standardised protocol. Lancet Diabetes Endocrinol 2015; 3: 296-303

[24] Laurent I, Astere M, Zheng F et al. Adrenal venous sampling with or without adrenocorticotropic hormone stimulation: A meta-analysis. J Clin Endocrinol Metab 2019; 104: 1060-1068

[25] Choi M, Scholl UI, Yue P et al. K+ channel mutations in adrenal aldosterone-producing adenomas and hereditary hypertension. Science 2011; 331: 768-772

[26] Azizan EA, Poulsen H, Tuluc P et al. Somatic mutations in ATP1A1 and CACNA1D underlie a common subtype of adrenal hypertension. Nat Genet 2013; 45: 1055-1060

[27] Scholl UI, Goh G, Stolting G et al. Somatic and germline CACNA1D calcium channel mutations in aldosterone-producing adenomas and primary aldosteronism. Nat Genet 2013; 45: 1050-1054

[28] Beuschlein F, Boulkroun S, Osswald A et al. Somatic mutations in ATP1A1 and ATP2B3 lead to aldosterone-producing adenomas and secondary hypertension. Nat Genet 2013; 45: 440-444, 444e1-2

[29] Nanba K, Omata K, Gomez-Sanchez CE et al. Genetic characteristics of aldosterone-producing adenomas in blacks. Hypertension 2019; 73: 885-892

[30] Nanba K, Omata K, Else T et al. Targeted molecular characterization of aldosterone-producing adenomas in white Americans. J Clin Endocrinol Metab 2018; 103: 3869-3876 
[31] Monticone S, Castellano I, Versace K et al. Immunohistochemical, genetic and clinical characterization of sporadic aldosterone-producing adenomas. Molecular and Cellular Endocrinology 2015; 411: 146-154

[32] Lenzini L, Rossitto G, Maiolino G et al. A meta-analysis of somatic $\mathrm{KCNJ} 5 \mathrm{~K}(+)$ channel mutations in 1636 patients with an aldosterone-producing adenoma. J Clin Endocrinol Metab 2015; 100: E1089-E1095

[33] Yamazaki Y, Omata K, Tezuka Y et al. Tumor cell subtypes based on the intracellular hormonal activity in KCNJ5-mutated aldosterone-producing adenoma. Hypertension 2018; 72: 632-640

[34] Ono Y, Yamazaki Y, Omata K et al. Histological characterization of aldosterone-producing adrenocortical adenomas with different somatic mutations. J Clin Endocrinol Metab 2020; 105: pii: dgz235, DOI: 10.1210/clinem/dgz235

[35] Kitamoto T, Suematsu S, Yamazaki Y et al. Clinical and steroidogenic characteristics of aldosterone-producing adenomas with ATPase or CACNA1D gene mutations. J Clin Endocrinol Metab 2016; 101: 494-503

[36] Inoue K, Yamazaki Y, Kitamoto T et al. Aldosterone suppression by dexamethasone in patients with $\mathrm{KCNJ5}$-mutated aldosterone-producing adenoma. J Clin Endocrinol Metab 2018; 103: 3477-3485

[37] Ono Y, Nakamura Y, Maekawa T et al. Different expression of 11 beta-hydroxylase and aldosterone synthase between aldosterone-producing microadenomas and macroadenomas. Hypertension 2014; 64: 438-444

[38] Tezuka Y, Yamazaki Y, Kitada M et al. 18-Oxocortisol synthesis in aldosterone-producing adrenocortical adenoma and significance of KCNJ5 mutation status. Hypertension 2019; 73: 1283-1290

[39] Nakamura Y, Kitada M, Satoh F et al. Intratumoral heterogeneity of steroidogenesis in aldosterone-producing adenoma revealed by intensive double- and triple-immunostaining for CYP11B2/B1 and CYP17. Mol Cell Endocrinol 2016; 422: 57-63

[40] Williams TA, Peitzsch M, Dietz AS et al. Genotype-specific steroid profiles associated with aldosterone-producing adenomas. Hypertension 2016; 67: 139-145

[41] Nanba K, Blinder AR, Rege J et al. Somatic CACNA1H mutation as a cause of aldosterone-producing adenoma. Hypertension 2020; 75: 645-649

[42] Wannachalee T, Zhao L, Nanba K et al. Three discrete patterns of primary aldosteronism lateralization in response to cosyntropin during adrenal vein sampling. J Clin Endocrinol Metab 2019; 104: 5867-5876

[43] Stowasser M, Bachmann AW, Tunny TJ et al. Production of 18-oxo-cortisol in subtypes of primary aldosteronism. Clin Exp Pharmacol Physiol 1996; 23: 591-593

[44] Mosso L, Gomez-Sanchez CE, Foecking MF et al. Serum 18-hydroxycortisol in primary aldosteronism, hypertension, and normotensives. Hypertension 2001; 38: 688-691

[45] Gordon RD, Hamlet SM, Tunny T] et al. Distinguishing aldosterone-producing adenoma from other forms of hyperaldosteronism and lateralizing the tumour pre-operatively. Clin Exp Pharmacol Physiol 1986; 13: 325-328

[46] Chu MD, Ulick S. Isolation and identification of 18-hydroxycortisol from the urine of patients with primary aldosteronism. J Biol Chem 1982; 257: 2218-2224

[47] Ulick S, Blumenfeld JD, Atlas SA et al. The unique steroidogenesis of the aldosteronoma in the differential diagnosis of primary aldosteronism. J Clin Endocrinol Metab 1993; 76: 873-878

[48] Hamlet SM, Gordon RD, Gomez-Sanchez CE et al. Adrenal transitional zone steroids, 18-oxo and 18-hydroxycortisol, useful in the diagnosis of primary aldosteronism, are ACTH-dependent. Clin Exp Pharmacol Physiol 1988; 15: 317-322
[49] Gomez-Sanchez CE, Montgomery M, Ganguly A et al. Elevated urinary excretion of 18-oxocortisol in glucocorticoid-suppressible aldosteronism. J Clin Endocrinol Metab 1984; 59: 1022-1024

[50] Satoh F, Morimoto R, Ono Y et al. Measurement of peripheral plasma 18-oxocortisol can discriminate unilateral adenoma from bilateral diseases in patients with primary aldosteronism. Hypertension 2015; 65: 1096-1102

[51] Eisenhofer G, Dekkers T, Peitzsch M et al. Mass spectrometry-based adrenal and peripheral venous steroid profiling for subtyping primary aldosteronism. Clin Chem 2016; 62: 514-524

[52] Zheng FF, Zhu LM, Nie AF et al. Clinical characteristics of somatic mutations in Chinese patients with aldosterone-producing adenoma. Hypertension 2015; 65: 622-628

[53] Taguchi R, Yamada M, Nakajima Y et al. Expression and mutations of KCNJ5 mRNA in Japanese patients with aldosterone-producing adenomas. J Clin Endocrinol Metab 2012; 97: 1311-1319

[54] Akerstrom T, Crona J, Delgado Verdugo A et al. Comprehensive re-sequencing of adrenal aldosterone producing lesions reveal three somatic mutations near the $\mathrm{KCN} 55$ potassium channel selectivity filter. PloS one 2012; 7: e41926

[55] Boulkroun S, Beuschlein F, Rossi GP et al. Prevalence, clinical, and molecular correlates of $\mathrm{KCN} J 5$ mutations in primary aldosteronism. Hypertension 2012; 59: 592-598

[56] Azizan EA, Murthy M, Stowasser $M$ et al. Somatic mutations affecting the selectivity filter of KCNJ5 are frequent in 2 large unselected collections of adrenal aldosteronomas. Hypertension 2012; 59: 587-591

[57] Monticone S, Hattangady NG, Nishimoto K et al. Effect of KCNJ5 mutations on gene expression in aldosterone-producing adenomas and adrenocortical cells. J Clin Endocrinol Metab 2012; 97: E1567E1572

[58] Azizan EA, Lam BY, Newhouse S] et al. Microarray, qPCR, and KCN]5 sequencing of aldosterone-producing adenomas reveal differences in genotype and phenotype between zona glomerulosa- and zona fasciculata-like tumors. J Clin Endocrinol Metab 2012; 97: E819-E829

[59] Omata K, Satoh F, Morimoto R et al. Cellular and genetic causes of idiopathic hyperaldosteronism. Hypertension 2018; 72: 874-880

[60] Arlt W, Lang K, Sitch A et al. Steroid metabolome analysis reveals prevalent glucocorticoid excess in primary aldosteronism. https:// www.ncbi.nlm.nih.gov/pubmed/28422753. JCl Insight 2017; 2: pii: 93136. doi: 10.1172/jci.insight.93136. eCollection 2017 Apr 20

[61] El Ghorayeb N, Mazzuco TL, Bourdeau I et al. Basal and Post-ACTH aldosterone and its ratios are useful during adrenal vein sampling in primary aldosteronism. J Clin Endocrinol Metab 2016; 101: 1826-1835

[62] Takeda M, Yamamoto K, Akasaka $\mathrm{H}$ et al. Clinical characteristics and postoperative outcomes of primary aldosteronism in the elderly. J Clin Endocrinol Metab 2018; 103: 3620-3629

[63] Wolley M], Gordon RD, Ahmed AH et al. Does contralateral suppression at adrenal venous sampling predict outcome following unilateral adrenalectomy for primary aldosteronism? A retrospective study. J Clin Endocrinol Metab 2015; 100: 1477-1484

[64] Tagawa M, Ghosn M, Wachtel $\mathrm{H}$ et al. Lateralization index but not contralateral suppression at adrenal vein sampling predicts improvement in blood pressure after adrenalectomy for primary aldosteronism. J Hum Hypertens 2017; 31: 444-449

[65] Turcu AF, Wannachalee T, Tsodikov A et al. Comprehensive analysis of steroid biomarkers for guiding primary aldosteronism subtyping. Hypertension 2020; 75: 183-192 